\title{
A New Fast Iterative Blind Deconvolution Algorithm
}

\author{
Mamdouh F. Fahmy ${ }^{1}$, Gamal M. Abdel Raheem ${ }^{1}$, Usama S. Mohamed ${ }^{1}$, Omar F. Fahmy ${ }^{2}$ \\ ${ }^{1}$ Department Electrical Engineering, Assiut University, Assiut, Egypt; ${ }^{2}$ Department of Electrical Engineering, Future University, \\ Cairo, Egypt. \\ Email: omarfarouk_mamdouh@hotmail.com
}

Received December $8^{\text {th }}, 2011$; revised January $9^{\text {th }}, 2012$; accepted January $18^{\text {th }}, 2012$

\begin{abstract}
Successful blind image deconvolution algorithms require the exact estimation of the Point Spread Function size, PSF. In the absence of any priori information about the imagery system and the true image, this estimation is normally done by trial and error experimentation, until an acceptable restored image quality is obtained. This paper, presents an exact estimation of the PSF size, which yields the optimum restored image quality for both noisy and noiseless images. It is based on evaluating the detail energy of the wave packet decomposition of the blurred image. The minimum detail energies occur at the optimum PSF size. Having accurately estimated the PSF, the paper also proposes a fast double updating algorithm for improving the quality of the restored image. This is achieved by the least squares minimization of a system of linear equations that minimizes some error functions derived from the blurred image. Moreover, a technique is also proposed to improve the sharpness of the deconvolved images, by constrained maximization of some of the detail wavelet packet energies. Simulation results of several examples have verified that the proposed technique manages to yield a sharper image with higher PSNR than classical approaches.
\end{abstract}

Keywords: Blind Image Deconvolution; Image Enhancement

\section{Introduction}

The goal of blind deconvolution is to recover two convolved signals $f$ and $h$ from their convolved (and normally noisy), version $g$. Neither $f$ nor $h$ is known. In image processing, $f$ represents the true image, whereas $h$ represents the Point Spread Function PSF, which is responsible for blurring $f$. Even if we have a priori information about the PSF, recovering the original image by inverse filtering is usually counterproductive, as it involves noise amplification, [1,2]. At this point it is worth mentioning that, not all blurring causes can be precisely determined. In general, the PSF can be described by the 2D Gaussian as in atmospheric turbulence or by circular PSF as in defocusing effects [3]. However, Central limit theorem implies that receiving blurred version of the image is closer to blurring the original image by a Gaussian distribution PSF. Note that the Gaussian PSF is one of the most difficult cases to deal with in blind deconvolution, as it can be factored into two Gaussian PSF. In the noiseless case $g=h^{* *} f$. Solution starts by choosing an initial guess of $f$, (normally taken to be the blurred image itself), then obtain $h$ as the least squares solution of $\left\|g-h^{* *} f\right\|$. Iteration is reversed and we seek to estimate $f$ using the estimated $h$. However, as the size of $h$ is much smaller than $f$, this approach is computationally prohibitive. Many efficient techniques have been proposed to solve this problem, [4-9]. In [4], an Iterative Blind Deconvolution technique $I B D$ has been proposed by alternate updating the 2-D FFT of $f$ and $h$, until the relation $G\left(\omega_{1}, \omega_{2}\right) \cong F\left(\omega_{1}, \omega_{2}\right) H\left(\omega_{1}, \omega_{2}\right)$, is almost satisfied. In its initial versions, it suffered from poor convergence, yet in latter versions [5], its robustness to noise and convergence properties are highly improved. An alternate double iteration algorithm that has good anti-noise capability has also been described in [6, 7]. It is known as the Richards-Lucy algorithm, and is characterized with robustness to either Poisson or Gaussian noise, [8,9]. In [5-10], a thorough treatment of different blind deconvolution techniques can be found. All these techniques require an exact estimation of the blurring PSF size. In view of the absence of any priori information about the PSF size, the application of the IBD, Richards-Lucy or any other blind deconvolution algorithms will fail to yield good quality restored images. This paper, addresses this problem. It shows how the optimum blurring size, can be accurately estimated for both noisy and noiseless images. Then, using this estimated PSF size the performance of IBD, RL or any other blind deconvolution algorithm is further improved. This improvement is achieved by iterating between updating the restored image $\hat{f}(m, n)$ and the PSF $\hat{h}(m, n)$ that minimizes some arbitrary largest absolute error devia- 
tions of the error $e(m, n)=g(m, n)-\hat{g}(m, n)$ where $\hat{g}=\hat{h} \otimes \hat{f}$. Further, the paper also describes a technique for improving the sharpness of the deconvolved image, through maximizing some of the wavelet packet detail energies, while minimizing the residual reconstruction error energy. Simulation results of several images blurred by Gaussian or circular PSF, have verified that the proposed techniques substantially improve the quality of the restored images.

\section{The Fast Iterative Blind Deconvolution Algorithm}

\subsection{Mathematical Preliminaries}

If the original image $f$ of size $M \times N$ is blurred by an unknown transfer function $h$ of size $J \times K$, then the blurred image $g$ is computed as

$$
g(m, n)=\sum_{k=0}^{J-1} \sum_{j=0}^{K-1} h(k, j) f(m-k, n-j)+w(m, n)
$$

$w(m, n)$ is the associated zero-mean additive noise. For simplicity, let $M=N, \quad J=K=N_{p}$. Using circular convolution properties, overlap is avoided if each row vectors of $f, g$ and $w$ is padded by zeros to make its length equals $M_{0}$, where $M_{0}=M+N_{p}-1=N_{0}$. So, if $f, g$ and $w$ represent $M_{0} N_{0} \times 1$ column vectors formed by stacking the rows of the extended matrices, Equation (1) can be expressed as

$$
g=H f+w
$$

$H$ is the block circulant $M_{0} N_{0} \times M_{0} N_{0}$ matrix, defined by

$$
\begin{aligned}
& H=\left[\begin{array}{cccc}
H_{0} & H_{M_{0}-1} & \ldots & H_{1} \\
H_{1_{1}} & H_{0} & \ldots & H_{2} \\
. & & & \\
\ldots . . & & & \\
H_{N_{0}-1} & H_{N_{0}-2} & \ldots & H_{0}
\end{array}\right] \\
& H_{j}=\left[\begin{array}{cccc}
h_{e}(j, 0) & h_{e}\left(j, N_{0}-1\right) & \ldots . & h_{e}(j, 1) \\
h_{e}(j, 1) & h_{e}(j, 0) & \ldots . & h_{e}(j, 2) \\
. & & & \ldots . \\
\ldots . & & & \\
h_{e}\left(j, N_{0}-2\right) & h_{e}\left(j, N_{0}-1\right) & \ldots & h_{e}(j, 0)
\end{array}\right]
\end{aligned}
$$

Equation (2), suggests that, $f$ can be recovered as

$$
f=\left(H^{t} H\right)^{-1} H^{t}(g-w)
$$

This equation indicates that even in case of prior knowledge of $h$ and $w$, the inverse of $\left(H^{t} H\right)$ apart from requiring huge amount of computation for ordinary sized images, can result in an unbounded per- turbation in the solution $f$. This problem is solved by taking the 2-D FFT of both $h$ and $f$, as is explained in the next section.

\subsection{The Constrained Lest Squares Error Algorithm}

The constrained least squared error algorithm [1,2], uses the 2-D FFT techniques, to obtain the restored image. It aims to obtaining a restored image $\hat{f}(m, n)$ that is the solution of the following constrained optimization problem: Find the optimum $\hat{f}(m, n), \hat{h}(m, n)$ that minimize the objective function $J$,

Minimize

$$
J=\left|Q\left(\omega_{1}, \omega_{2}\right) \hat{F}\left(\omega_{1}, \omega_{2}\right)\right|^{2} \text { for all }-\pi \leq\left(\omega_{1}, \omega_{2}\right) \leq \pi
$$

Subject to

$$
\begin{gathered}
\left|G\left(\omega_{1}, \omega_{2}\right)-\hat{H}\left(\omega_{1}, \omega_{2}\right) F\left(\omega_{1}, \omega_{2}\right)\right|^{2} \leq \varepsilon^{2} \\
\hat{H}\left(\omega_{1}, \omega_{2}\right)=\sum_{m=0}^{M_{1}-1 N_{1}-1} \hat{n} \hat{h}(m, n) \mathrm{e}^{-j\left(m \omega_{1}+n \omega_{2}\right)}, \\
\hat{F}\left(\omega_{1}, \omega_{2}\right)=\sum_{m=0}^{M_{2}-1 N_{2}-1} \hat{f}(m, n) \mathrm{e}^{-j\left(m \omega_{1}+n \omega_{2}\right)}
\end{gathered}
$$

Using the Lagrange multiplier technique, this problem can be formulated as

\section{Minimize}

$$
\begin{aligned}
J & =\left|Q\left(\omega_{1}, \omega_{2}\right) \hat{F}\left(\omega_{1}, \omega_{2}\right)\right|^{2} \\
& +\lambda\left(\left|G\left(\omega_{1}, \omega_{2}\right)-\hat{H}\left(\omega_{1}, \omega_{2}\right) \hat{F}\left(\omega_{1}, \omega_{2}\right)\right|^{2}\right)-\varepsilon^{2}
\end{aligned}
$$

The solution to this constrained minimization problem, can be shown to be

$$
\hat{F}\left(\omega_{1}, \omega_{2}\right)=\frac{\lambda \hat{H}^{*}\left(\omega_{1}, \omega_{2}\right) G\left(\omega_{1}, \omega_{2}\right)}{\left|Q\left(\omega_{1}, \omega_{2}\right)\right|^{2}+\lambda\left|\hat{H}\left(\omega_{1}, \omega_{2}\right)\right|^{2}}
$$

Now, the function $Q\left(\omega_{1}, \omega_{2}\right)$ is chosen to boost the high frequency energies of $F\left(\omega_{1}, \omega_{2}\right)$. As all natural images have pre-dominant low frequency content, minimizing $J$ means that the true image $f(m, n)$ is obtained, or at least nearly obtained. $Q$ can be chosen in many different ways. In [1], two formulas were given to $q(m, n)$ to approximate Laplacian function. In this paper, a simpler of $Q\left(\omega_{1}, \omega_{2}\right)$ that satisfies the high frequency emphasis requirements, is proposed. It is chosen as

$Q\left(\omega_{1}, \omega_{2}\right)=\frac{1}{\hat{F}\left(\omega_{1}, \omega_{2}\right)}$. Using this choice, leads to the following iterative restoration algorithm

$$
\hat{F}\left(\omega_{1}, \omega_{2}\right)=\frac{H^{*}\left(\omega_{1}, \omega_{2}\right)}{\frac{\alpha}{\hat{F}^{(k-1)}\left(\omega_{1}, \omega_{2}\right)}+\left|H\left(\omega_{1}, \omega_{2}\right)\right|^{2}} \quad \alpha=\frac{1}{\lambda}
$$


This is precisely the update algorithm cited without proof in [4]. There, it is proposed to apply this update formula to estimate $\hat{H}\left(\omega_{1}, \omega_{2}\right)$. This results in the so-called Iterative Blind Deconvolution algorithm, $I B D$. It is an improved version of the original Iterative Deconvolution described [3], and overcome many of its shortcomings. This algorithm is implemented using the MatLab function deconvblind.

Now, the success of the IBD algorithm, as well as many other iterative deconvolution algorithms in estimating the original images depends on the precise estimation of the PSF order. The next section shows how this order is precisely estimated, in view of no priori information about the PSF order.

\subsection{PSF Size Estimation}

All iterative blind deconvolution algorithms require an estimate of the PSF size. To our knowledge, this is done on trial and error basis until good quality restored image is obtained. An analytical method is now given to estimate the optimum PSF size.

To analyze this problem, let the estimated PSF and image, be

$$
\begin{aligned}
& \hat{h}(m, n)=h(m, n)+\delta h(m, n), \\
& \hat{f}(m, n)=f(m, n)+\delta f(m, n)
\end{aligned}
$$

where $f(m, n)$ and $h(m, n)$ are the original image and the true PSF filter. Note that in all blind deconvolution algorithms, $\delta h(m, n)$ controls $\delta f(m, n)$. So, the blurred received image $g(m, n)$, is given by

$$
\begin{aligned}
& g(m, n)=\hat{h}(m, n) * * \hat{f}(m, n) \\
& =(h(m, n)+\delta h(m, n)) * *(f(m, n)+\delta f(m, n)) \\
& =h(m, n) * * f(m, n)+e(m, n)
\end{aligned}
$$

Clearly, due to the uncertainty of $h(m, n), e(m, n)$ can be considered as additive noise. It mainly affects the high frequency energy bands of the image. As the perturbation $\delta h(m, n)$ gets smaller, their energy contribution to $e(m, n)$ becomes smaller. This suggests to decompose $e(m, n)$ using $n$-level wavelet packet decomposition and compute the detail energy in the last (high) wavelet packet $W P_{n}$.

If the blurred image is contaminated with zero mean AWGN, then the blurred noisy image has to be de-noised prior to PSF order estimation. The threshold level is computed as, [11]

$$
T=\sigma \sqrt{2 \log _{e} N}
$$

$\sigma^{2}$ is the variance of the $W P_{n}$. It is determined through estimating its $p d f$ distribution as described in [12]. $N$ is its length when converted to a column vector. The fol- lowing example, illustrates PSF estimation in both the noiseless and noisy cases, for Gaussian and circular defocusing blurring filters

\section{Ex. 1:}

The proposed PSF order estimation method is verified by the following simulations. The test images used, are blurred using $8 \times 8$ Gaussian filter with $\sigma^{2}=10$, and circular averaging filter (pillbox), with radius $r=3$. For an arbitrary PSF order, the program estimates the deblurred image, using the Matlab function deconvblind, or deconvlucy. The parameters of these algorithms are: Number of cycles $=40$, Threshold $\varepsilon=0.005$, The error signal $e(m, n)$ is decomposed using 2-level "sym4" wavelet decomposition. Figure 1 shows the behavior of the detail energy of the $H H$ sub-band with different PSF order, for these blurred images. This precise PSF estimation subsequently leads to a significant improvement restored image quality, as will be shown in the following section.

In the noisy case, the blurred image is contaminated with zero mean AWGN of $\sigma^{2}=0.01$. The blurred noisy image variances are [0.0557 0.04210 .0265$]$, respectively. In order to de-noise this noisy image, it is decomposed with 2-level "sym4" wavelet decomposition. The probability distribution function $p d f$, of the last $H H$ wavelet packet is computed using the Bspline pdf estimation technique proposed in [12], using 3-level cubic Bspline wavelet with 128 histogram bins. Figure 2, compares the $p d f$ of the $H H$ sub-band with Gaussian random variable distribution having the same mean and variance. It also shows detail energy performance for both Gaussian and circular blurring PSF. Again, this figure shows that apart from accurately estimating the $p d f$, it yields the optimum PSF size for further deblurring.

\subsection{The Proposed Fast Iterative Blind Deconvolution Algorithm}

The proposed Fast Iterative Blind Deconvolution algorithm FIBD, is initialized by estimating the PSF order, as described above using rough estimations of the original image provided by available algorithms, (like deconvblind, deconvlucy, $\cdots)$. Having estimated the blurring PSF order, the algorithm iterates between updating the restored image $\hat{f}(m, n)$ and the PSF $\hat{h}(m, n)$. The update is based on minimizing some arbitrary $M_{x}$ largest absolute error deviations of $e=|g-\widehat{h} * * \widehat{f}|$. The algorithm works in the spatial domain and is summarized as follows:

1) For the $k^{\text {th }}$ estimate, evaluate $\hat{g}=\widehat{h}_{k} * * \widehat{f}_{k}$. Evaluate $H$ from $\hat{h}$ as in Equations (3a) and 3(b). Evaluate the error $\Delta g=g-\hat{g}$. Arrange $\Delta g$ in a vector form.

2) Sort $|\Delta g|=|g-\hat{g}|$ in ascending order using the 

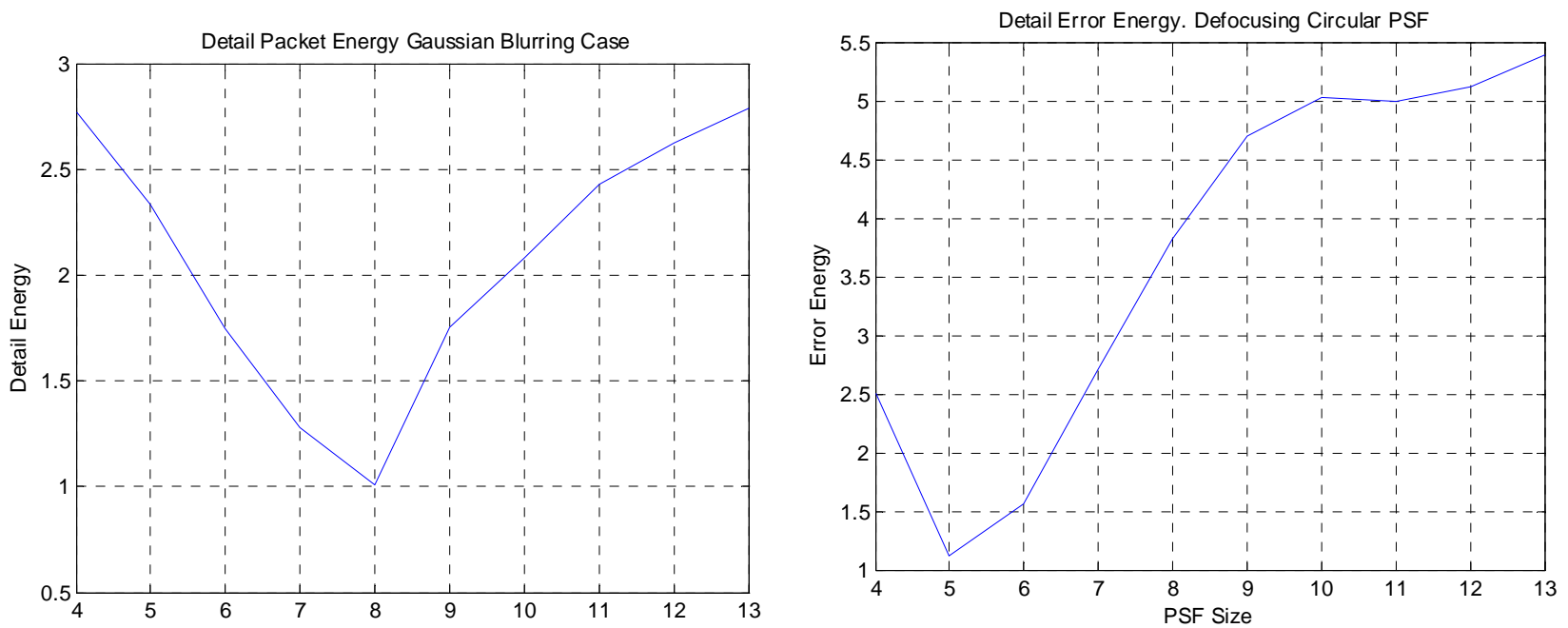

(a)
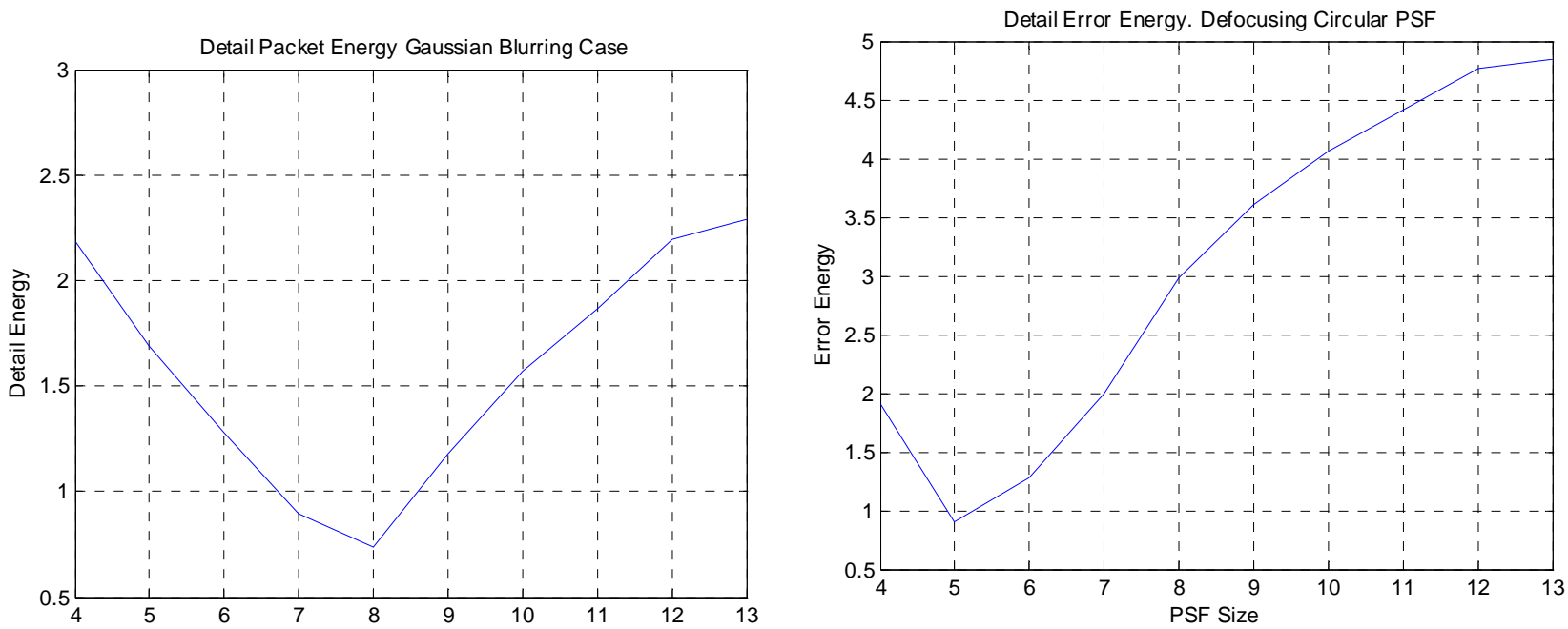

(b)
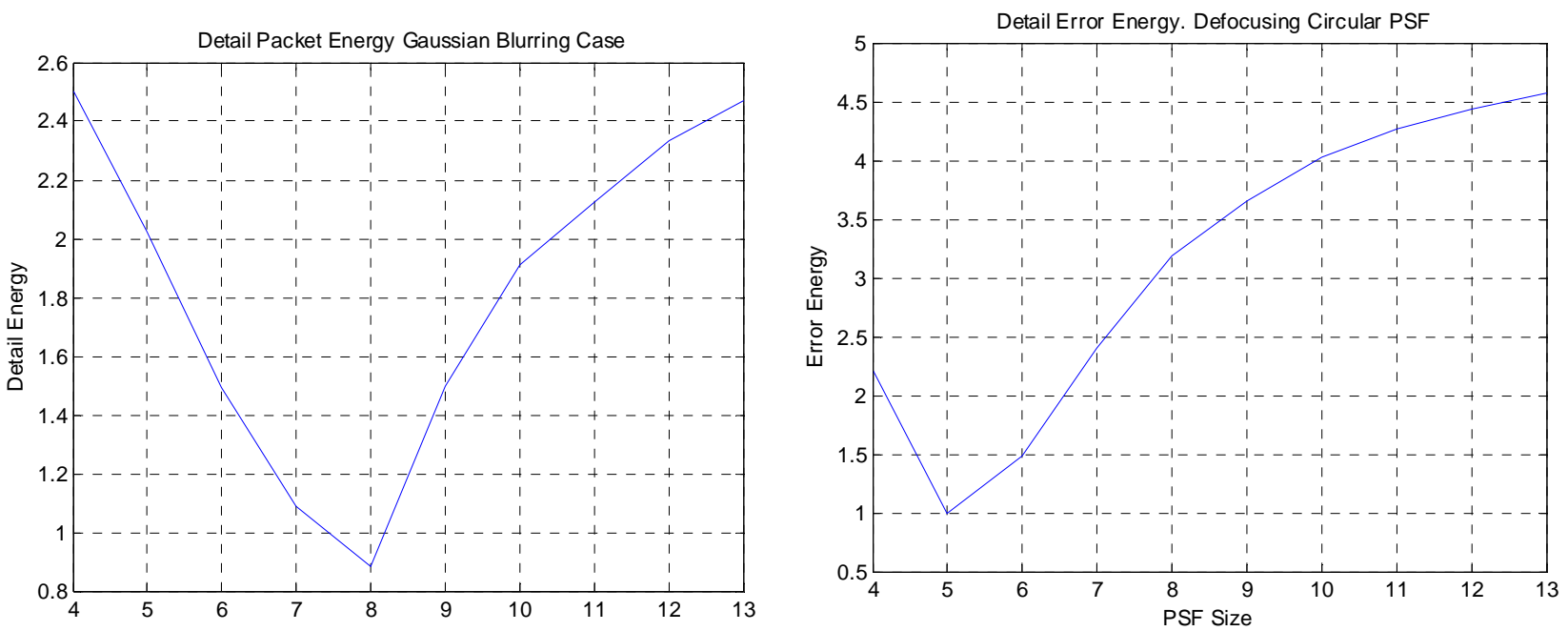

(c)

Figure 1. The detail energy of the $\mathrm{HH}$ sub-band for (a) Cameraman; (b) Lena; (c) Mandrill. 

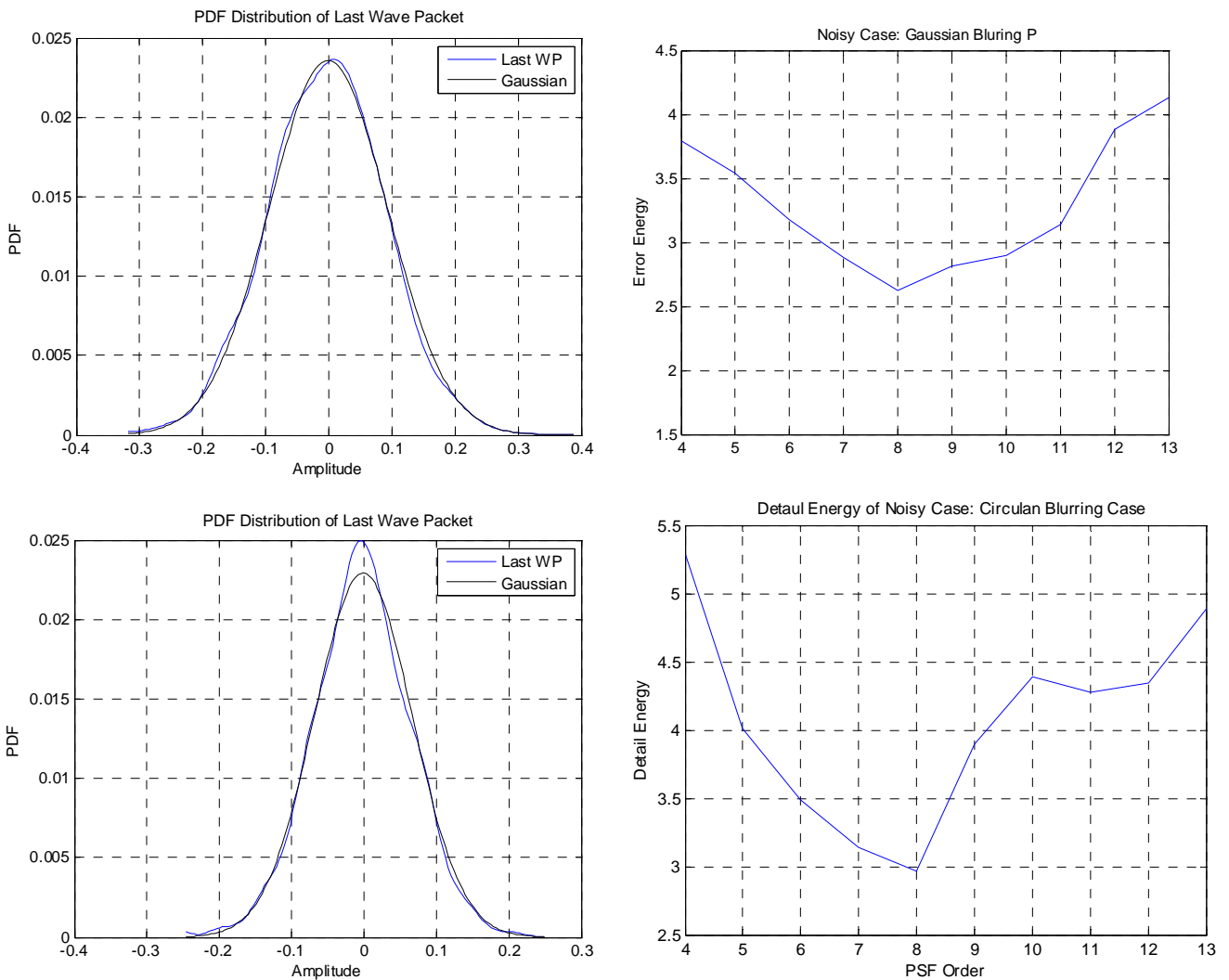

(a)
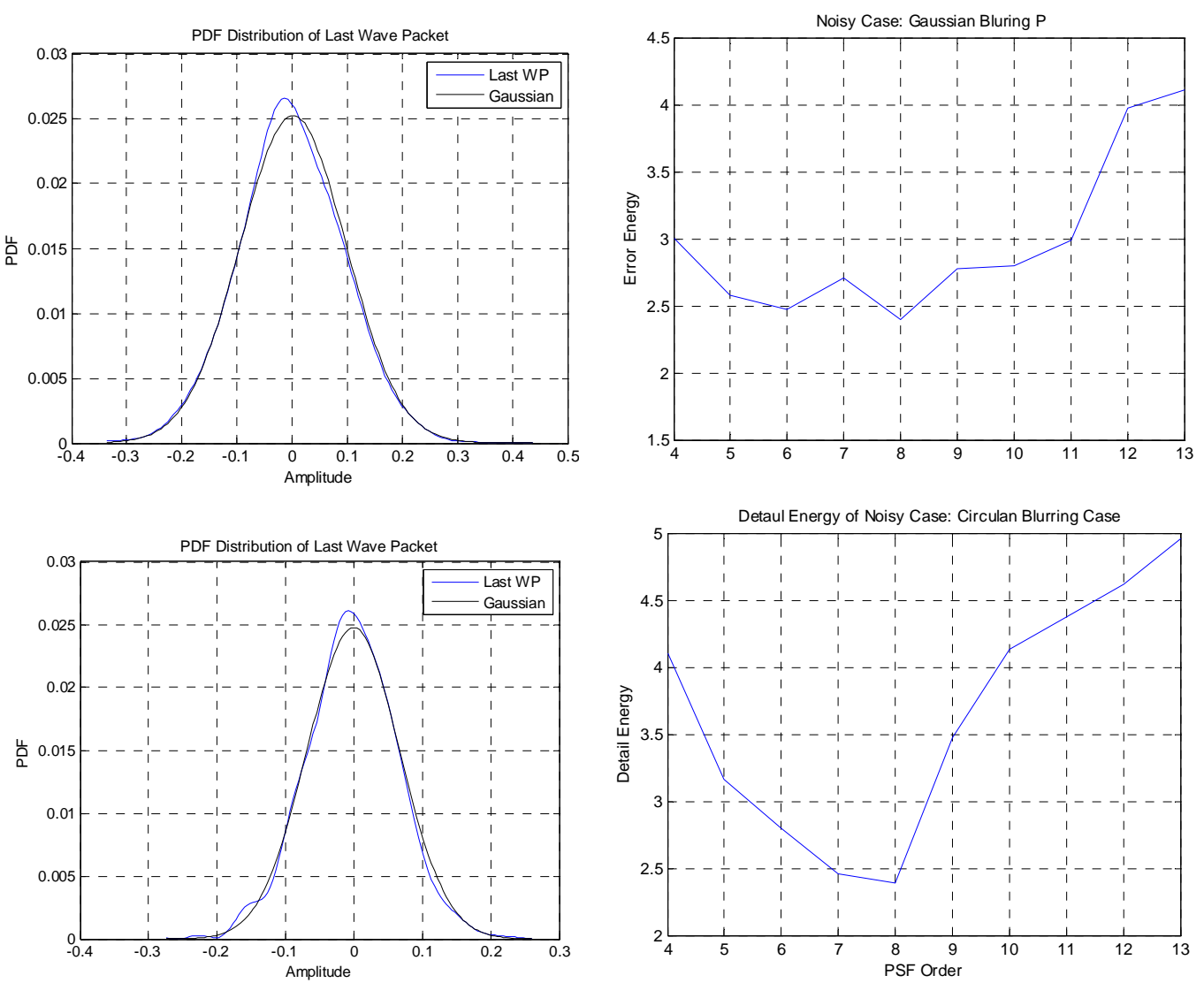

(b) 

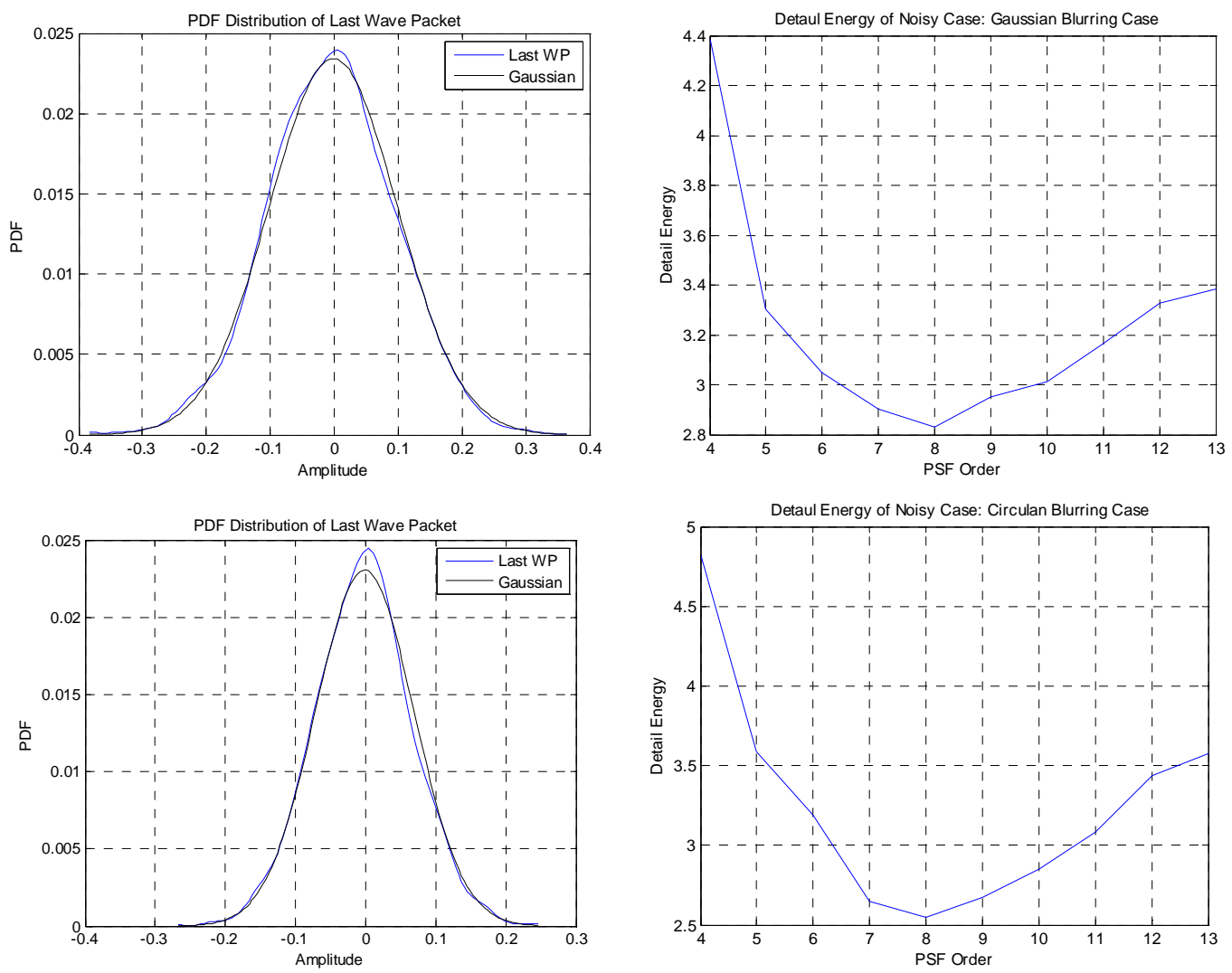

(c)

Figure 2. The pdf of the $\mathrm{HH}$ sub-band \& detail energy performance for both Gaussian and circular blurring PSF. (a) Cameraman; (b) Lena; (c) Mandrill.

Matlab command $\left[V_{x}, I_{x m}\right]=\operatorname{sort}(|\Delta g|)$. Subsequently, Sort $H^{(k)}$ as $H_{s}=H^{(k)}\left(I_{x m}, I_{x m}\right)$.

3) For a prescribed number $M_{x}$, pick the $M_{x}$ largest deviations of $M_{x}$. Denote by

$$
\begin{gathered}
\Delta g_{M_{x}}=\Delta g\left(I_{x m}\left(\text { end }-M_{x}+1: \text { end }\right)\right) . \\
\text { Partition } H_{s}=\left[\begin{array}{ll}
H_{11} & H_{12} \\
H_{21} & H_{22}
\end{array}\right] \uparrow M_{x} \\
\leftrightarrow M_{x}
\end{gathered}
$$

4) Only update $\hat{f}_{M_{x}}^{(k)}$ responsible for the $M_{x}$ largest deviations of $M_{x}$. The update increments together with the updated $\left|\Delta g_{M_{x}}\right|$, are given by

$$
\begin{gathered}
-\Delta g=\left[\begin{array}{l}
H_{12} \\
H_{22}
\end{array}\right] \Delta f_{M_{x}}^{(k)} \\
f^{(k+1)}=f^{(k)}+\left[\operatorname{zeros}\left(M_{0} N_{0}-M_{x}\right) ; \Delta f_{M_{x}}^{(k)}\right]
\end{gathered}
$$

This concludes the image restoration cycle.

Updating $\widehat{h}^{(k)}$ proceeds similarly through minimizing the energy of $\|g-\hat{g}\|$ using the updated image $\hat{f}^{(k+1)}(m, n)$. This update can easily be achieved either through minimizing the objective function $\zeta=\sum_{m} \sum_{n} e^{2}(m, n), e=g-\hat{h} * * \widehat{f}$ or more simply as the least squares solution of Equation (4), when $h$ and $f$ are interchanged.

Ex. 2:

The blurred images of Ex. (1), are deconvolved through minimizing the largest $M_{x}$ peak deviations of the absolute error of $e(m, n)$. Tables 1(a) and (b) compare the PSNR improvements of the proposed FIBD technique, over the standard deconvlucy $(R L)$ and deconvblind (IBD) algorithms for both $8 \times 8$ Gaussian and Circular PSF with $r=3$ filters.

These tables indicate that the proposed FIBD algorithm yields a significant PSNR improvement, over the standard RL and IBD algorithms. This is due to the fact that the algorithm modifies pixels responsible for the severest image degradation, unlike other methods using 2-D FFT that considers all parts of the image to have equal importance. Figures 3-5, show the rate of convergence as well as the deconvolved images, for the Gaussian blurring filter, whereas Figure 6 illustrated the blurring and deconvolved images in the circular blurring case. To end this section, it is worth pointing out that, if the PSF size is chosen different from the value estimated in the previous section, severe degradation of the quality of 
Table 1. (a) Gaussian PSF.

\begin{tabular}{cccccccc}
\hline & \multirow{2}{*}{$\mathrm{RL}$} & \multirow{2}{*}{ IBD } & \multicolumn{5}{c}{ FIBD } \\
\cline { 4 - 8 } & & & $M_{x}=64$ & 128 & 256 & 512 & 1024 \\
\hline Cameraman & 23.5290 & 23.6468 & 25.0114 & 25.1386 & 25.3356 & 25.5039 & 25.6131 \\
Lena & 24.8168 & 25.1177 & 25.6029 & 25.7005 & 25.8791 & 25.8991 & 26.1026 \\
Mandril & 22.0956 & 22.3211 & 23.1868 & 23.3061 & 23.3616 & 23.5978 & 23.5822 \\
\hline
\end{tabular}

Table 1. (b) Circular PSF (defocusing).

\begin{tabular}{|c|c|c|c|c|c|c|c|}
\hline & \multirow{2}{*}{ RL } & \multirow{2}{*}{ IBD } & \multicolumn{5}{|c|}{ FIBD } \\
\hline & & & $M_{x}=64$ & 128 & 256 & 512 & 1024 \\
\hline Cameraman & 25.7076 & 25.6617 & 26.3235 & 26.4553 & 26.6151 & 26.6542 & 26.6373 \\
\hline Lena & 27.2209 & 27.2219 & 27.5423 & 27.7395 & 27.8868 & 28.0509 & 28.0413 \\
\hline Mandril & 24.0190 & 23.7927 & 24.3658 & 24.4249 & 24.5135 & 24.6128 & 24.6197 \\
\hline
\end{tabular}

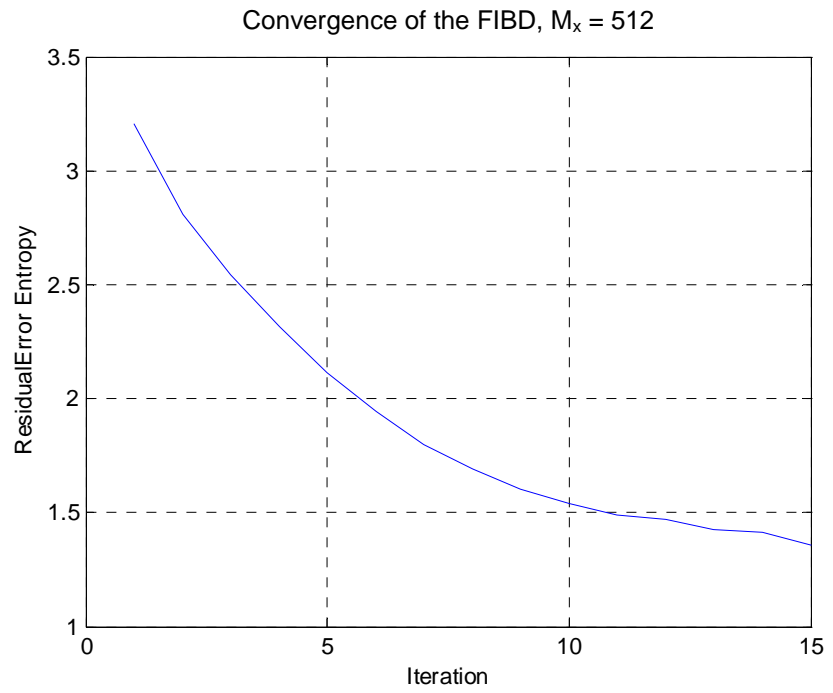

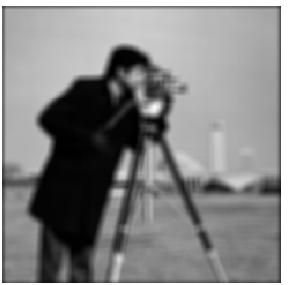

Blurred Image

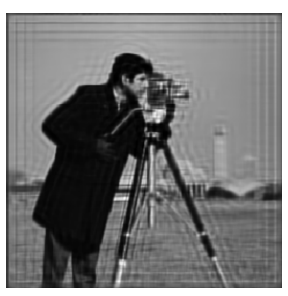

RL Image

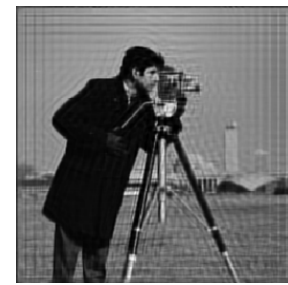

IBD Image

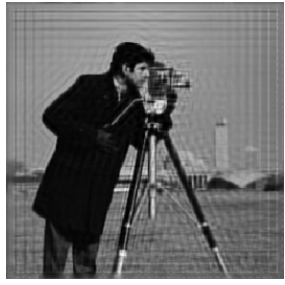

FIBD Image, $\mathrm{M}_{\mathrm{x}}=512$

Figure 3. The rate of convergence as well as the deconvolved Cameraman images for Gaussian case.

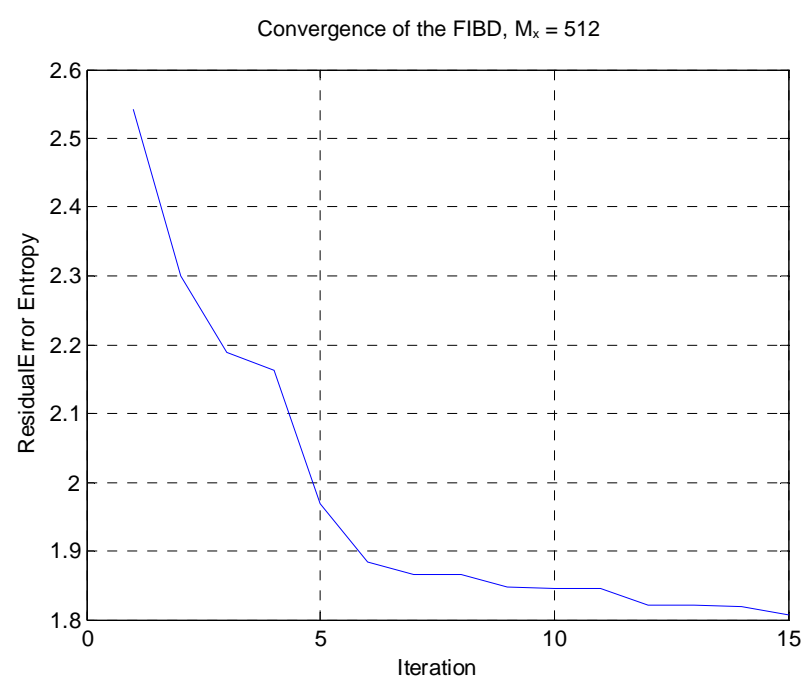

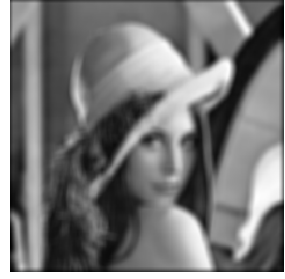

Blurred Image

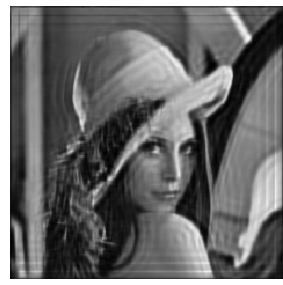

RL Image

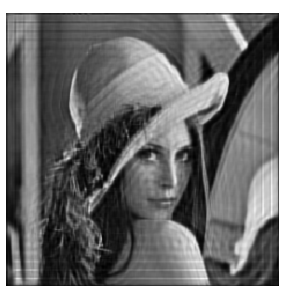

IBD Image

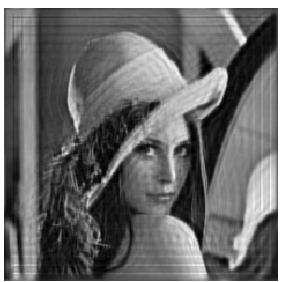

FIBD Image, $\mathrm{M}_{\mathrm{x}}=512$

Figure 4. The rate of convergence as well as the deconvolved Lena images for Gaussian case. 


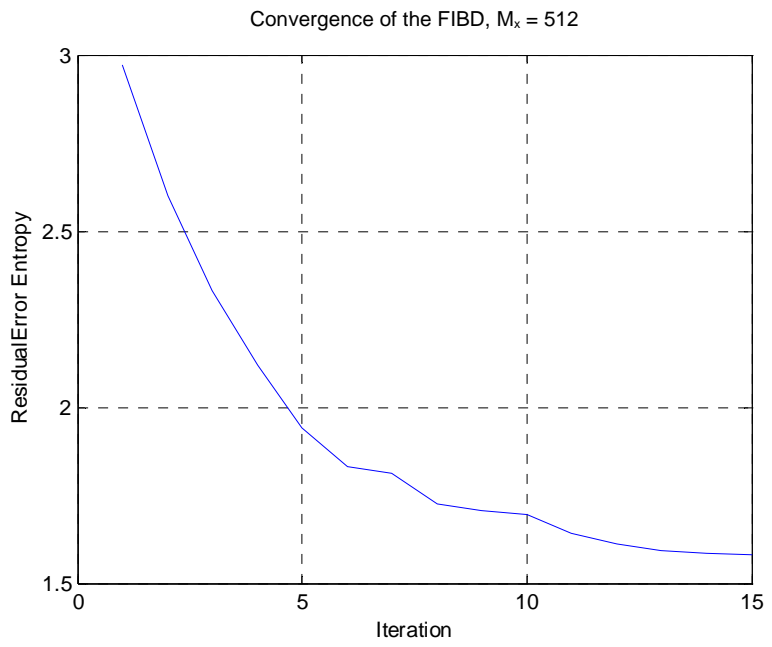

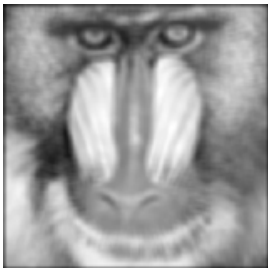

Blurred Image

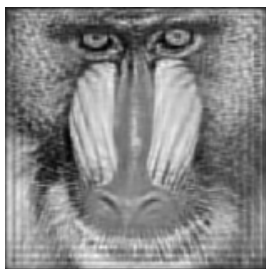

RL Image

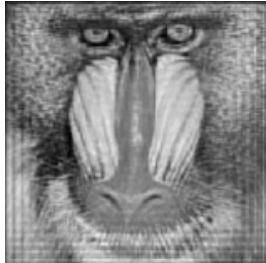

IBD Image

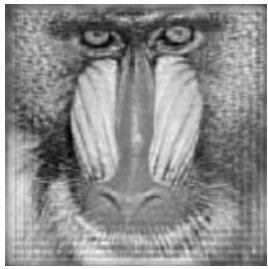

FIBD Image, $M_{x}=512$

Figure 5. The rate of convergence as well as the deconvolved Mandril images for Gaussian case.
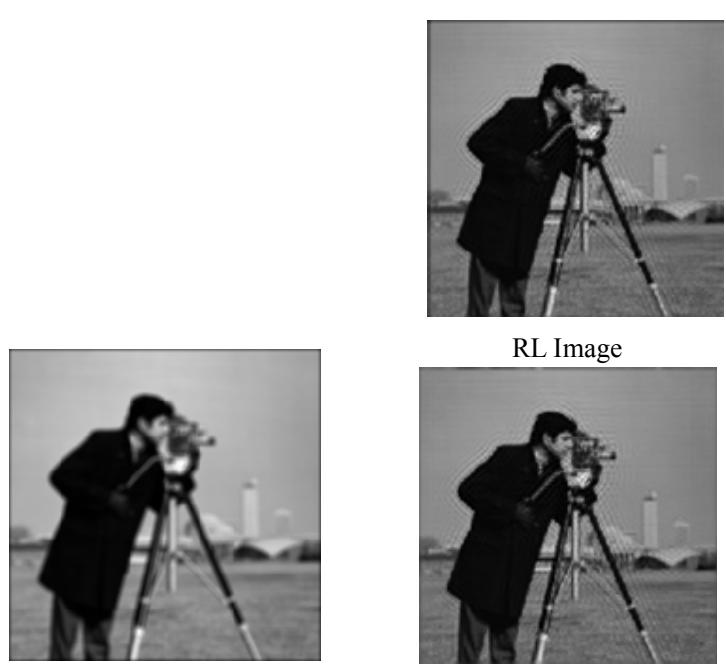

Circular Blurred Image

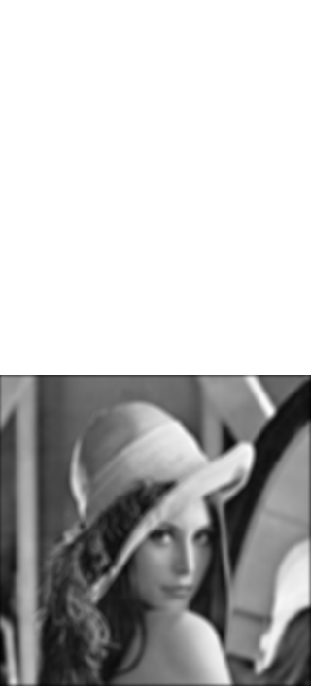

Circular Blurred Image
RL Image

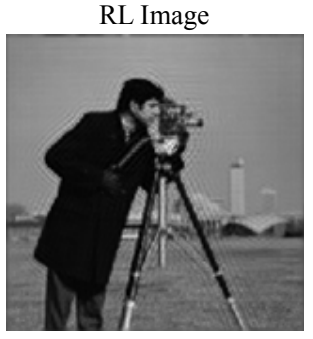

FIBD Image

(a)

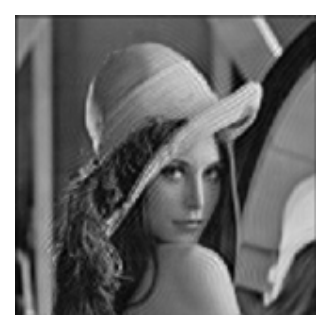

RL Image

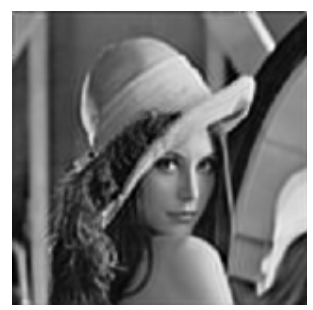

FIBD Image

(b)

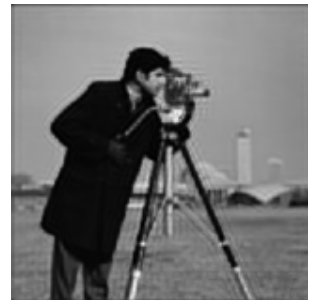

IBD Image

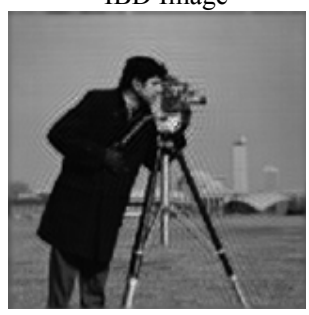

Wp Enhanced Image

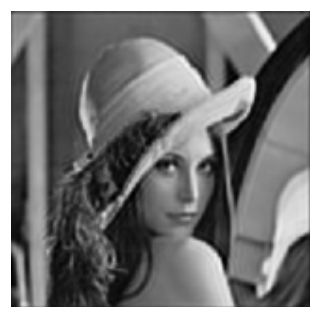

IBD Image

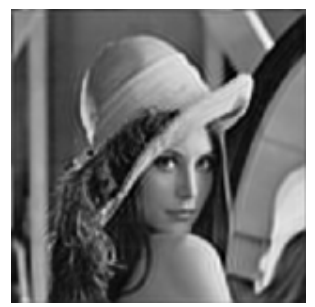

Wp Enhanced Image 


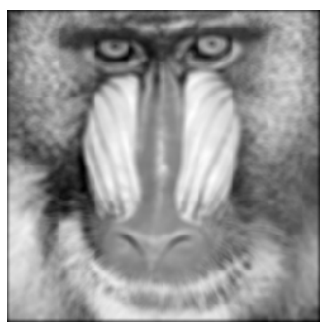

Circular Blurred Image

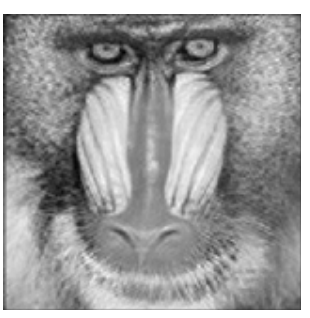

RL Image

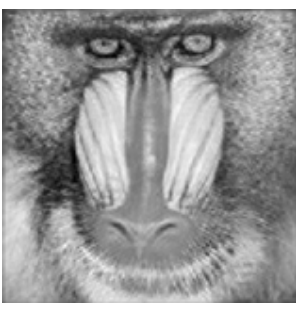

FIBD Image

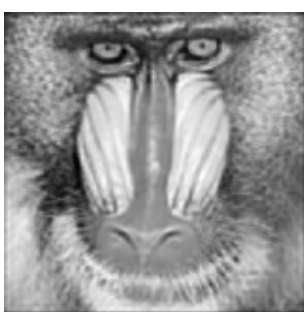

IBD Image

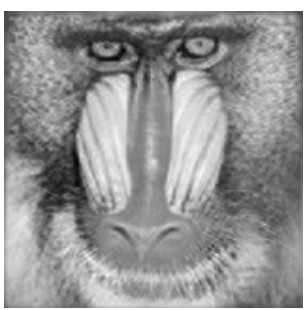

Wp Enhanced Image

(c)

Figure 6. The blurring and deconvolved images.

the deconvolved image occurs.

\section{Improving Sharpness of the Restoration Quality}

As blurring affects the energies of the high frequency bands of the image, it is clear that deblurring should boost these high frequency energies. To explain this idea, consider the Matlab Cameraman image that is blurred using a Gaussian PSF of size $8 \times 8$ and $\sigma^{2}=10$. Table 2 compares the percentage sub-band energies of 1-level "Sym4" wavelet decomposition, of the original Cameraman image, with those obtained for blurred, RL, IBD and the proposed FIBD deconvolved images. The FIBD image is obtained using $M_{x}=64$. This table indicates that natural images have a significant amount of energy concentration in its $L L$ sub-band. Moreover, blurring reduces the $L H, H L$ and $H H$ sub-bands. It also suggests that, the deblurred image quality can be improved through boosting the energies of the wavelet packets $L H, H L, H H$. This goal can be achieved by processing these sub-bands by a 2D mask $H_{0}$ that optimizes their energies while minimizing the residual energy of $J_{e r}$. In order to reduce the effects of noise amplifications that predominates the $\mathrm{HH}$ sub-band, only the coefficients of the $L H$ and $H L$ are modified. The steps of the optimization algorithm can be summarized as follows:

1) Apply the FIBD algorithm to determine the optimum deconvolved image $\hat{f}^{(k)}(m, n)$, for the received blurred image $g(m, n)$.

2) Obtain the 1-level "Sym4" wavelet packet decomposition of $\hat{f}$.

3) For an arbitrary 2-D matrix $H_{0}$, normalize it to be a unity variance matrix, (in order to avoid collapsing to zero in subsequent minimization steps). Filter the packets $L H$ and $H L$ coefficients with $H_{0}$. Then, reconstruct the image from its modified wavelet packet using the synthesis bank to get $y(m, n)$. Evaluate the residual error

$$
J_{e r}(m, n)=g(m, n)-\sum_{r=0}^{N_{1}-1} \sum_{l=0}^{N_{1}-1} \hat{h}(r, l) y(m-r, n-l)
$$

4) Using any unconstrained minimization algorithm, find the elements of $H_{0}$ that optimizes the detail packet energies while minimizing the error energy.

Tables 3(a) and (b) compare the PSNR improvements achieved with the classical IBD, FIBD as well as the Wave packet Optimized FIBD, using $M_{x}=64,128$, respectively when applying these steps to the above blurred images. In order to speed up computations, the 2-D mask $H_{0}$, is taken to be $4 \times 4$.

Edge improvements are checked by evaluating the norm of the difference between the exact edge of the original image, and the restored image edge. Table 4 compares the edge improvement of the optimized FIBD with the RL and IBD restorations for Gaussian blurring PSF. This table indicates that, coupled with the PSNR improvement, except for the Mandril image, the proposed FIBD and its optimized wavelet version are sharper than RL and IBD counterparts. Thus, one can conclude that FIBD and its optimized wavelet version provide a superior deblurring image restoration technique. Figures 6(a)-(c), compare the RL, IBD, FIBD and the optimized FIBD for the 3 Matlab images, with the FIBD technique for $M_{X}=64$.

\section{Conclusion}

This paper, describes how, in blind deconvolution when 
Table 2. \% Sub-band energy concentration.

\begin{tabular}{cccccc}
\hline WP & Original & Blurred & RL & IBD & FEIBD \\
\hline LL & 99.2777 & 99.9890 & 99.7106 & 99.5414 & 99.6324 \\
LH & 0.2204 & 0.0049 & 0.1230 & 0.1883 & 0.1403 \\
HL & 0.4264 & 0.0061 & 0.1652 & 0.2680 & 0.2250 \\
HH & 0.0755 & 0.0000 & 0.0012 & 0.0023 & 0.0023 \\
\hline
\end{tabular}

Table 3. (a) Wavelet packet PSNR improvement for Gaussian blurred images.

\begin{tabular}{cccccc}
\hline \multirow{2}{*}{ Blurring Type } & \multirow{2}{*}{ IBD } & \multicolumn{2}{c}{ FEIBD } & \multicolumn{2}{c}{ Opt. FEIBD } \\
\cline { 3 - 6 } & & 64 & 128 & 64 & 128 \\
\hline Cameraman & 23.6468 & 25.0114 & 25.1386 & 25.3257 & 25.3742 \\
Lena & 25.1177 & 25.6029 & 25.7005 & 25.4704 & 25.5538 \\
Mandril & 22,3211 & 23.1868 & 23.3061 & 23.3489 & 23.3914 \\
\hline
\end{tabular}

Table 3. (b) Wavelet packet PSNR improvement for circular blurred images.

\begin{tabular}{cccccc}
\hline \multirow{2}{*}{ Blurring Type } & \multirow{2}{*}{ IBD } & \multicolumn{3}{c}{ FEIBD } & \multicolumn{3}{c}{ Opt. FEIBD } \\
\cline { 3 - 6 } & & 64 & 128 & 64 & 128 \\
\hline Cameraman & 25.6617 & 26.3235 & 26.4553 & 26.3346 & 26.4336 \\
Lena & 27.2219 & 27.5423 & 27.7395 & 27.0878 & 27.1839 \\
Mandril & 23.7927 & 24.3658 & 24.4249 & 24.3802 & 24.4237 \\
\hline
\end{tabular}

Table 4. Edge error energy: Gaussian blurring.

\begin{tabular}{ccccc}
\hline \multirow{2}{*}{ Blurring Type } & \multirow{2}{*}{ RL } & IBD & \multicolumn{2}{c}{ WP Decomposition } \\
\cline { 4 - 5 } & & & 54 & 528 \\
\hline Cameraman & 53.6004 & 53.6656 & 49008 & 42.0000 \\
Lena & 52.4309 & 50.4480 & 49.6588 & 49.3254 \\
Mandril & 48.2804 & 48.4562 & 49.7896 \\
\hline
\end{tabular}

there is no priori information about both the true image and/or the blurring PSF, the size of the blurring PSF, can be accurately estimated for both noiseless and noisy blurred images. The paper also describes how using this estimated PSF size; the IBD or RL deconvolved images can be significantly improved. The proposed algorithms characterized by its fast convergence as a result of solving expressing pixels modifications as the solution of a set of linear equations. A novel method was also described to increase the sharpness of the deconvolved images. It remains to extend this work to the noisy case.

\section{REFERENCES}

[1] M. J. T. Smith and A. Docef, "A Study Guide for Digital Image Processing," Scientific Publishers Inc., Georgia, 1999.

[2] R. C. Gonzalez and R. E. Woods, "Digital Image Processing," 2nd Edition, Addison-Welsely Publishing Company, Reading, 1987.
[3] R. L. Lagendijk and J. Biemond, "Iterative Identification and Restoration of Images," Kluwer Academic Publishers, Boston, 1991. doi:10.1007/978-1-4615-3980-3

[4] G. R. Ayers and G. C. Danty, "Ierative Blind Deconvolution Method and Its applications," Optics Letters, Vol. 13, No. 7, 1988, pp. 547-549. doi:10.1364/OL.13.000547

[5] D. Kundur and D. Hatzinakios, "Blind Image Deconvolution Revisted," IEEE Magazine on Signal Processing, Vol. 13, No. 6, 1996, pp. 61-63.

[6] D. A. Fish, A. M. Brinicombe, E. R. Pike and J. G. Walker, "Blind Deconvolution by Means of the Richardson-Lucy Algorithm," Journal of the Optical Society of America A, Vol. 12, No. 1, 1995, pp. 58-65. doi:10.1364/JOSAA.12.000058

[7] D. S. C. Biggs and M. Andrews, "Acceleration of Iterative Image Restoration Algorithms," Applied Optics, Vol. 36, No. 8, 1997, pp. 1766-1775. doi:10.1364/AO.36.001766

[8] M. Jiang and G. Wang, "Development of Blind Image Deconvolution and Its Applications," Journal of X-ray 
Science and Ology, Vol. 11, 2003, pp. 13-19.

[9] H. Y. Liu, Y. S. Zhang and J. I. Song, "Study on the Methods of Super Resolution Image Reconstruction," The International Archives of the Photognanmetry, Remote Sensing and Spatial Information Science, Vol. XXVII, Part B2, Beijing, 2008.

[10] D. Kundur and D. Hatzinakios, "Blind Image Deconvolution," IEEE Magazine on Signal Processing, Vol. 13,
No. 3, 1996, pp. 43-64.

[11] S. Mallat, "A Wavelet Tour of Signal Processing, the Sparse Way," Academic Press, New York, 2009.

[12] M. F. Fahmy, G. M. Fahmy and O. M. Fahmy, "Bspline Wavelets in Signal De-Noising and Image Compression," Journal of Signal, Image and Video Processing, Vol. 5, No. 2, 2011, pp. 141-153.

doi:10.1007/s11760-009-0148-x 